\title{
Mormalización de la Calidad del Almidón Agrio de Yuca, a través de Mezclas de Almidones y de Raíces de Yuca
}

\author{
Irene Tischer* \\ Martín Moreno** \\ Mercedes Andrade***
}

* Ph.D. Profesora Titular de la Escuela de Ingeniería de Sistemas y Computación - Facultad de Ingeniería Universidad del Valle - Santiago de Cali - Colombia. e-mail:irene@eisc.univalle.edu.co

** Msc., Candidato a Doctor, Universidad Politécnica de Valencia - España. Profesor Titular Escuela de Ingeniería Mecánica - Universidad del Valle - Santiago de Cali - Colombia.

e-mail:mamoreno@univalle.edu.co

*** Msc. Profesora Asociada - Escuela de Ingeniería Industrial y Estadística - Universidad del Valle Santiago de Cali - Colombia.

e-mail:merandre2002@yahoo.com.mx

Fecha de recepción: Octubre 28 de 2002

Fecha de aprobación: Marzo 26 de 2003

\section{RESUMEN}

El almidón agrio de yuca se obtiene artesanalmente por fermentación natural de almidón nativo y se usa en la elaboración de productos tradicionales de panadería. Las propiedades esperadas se refieren a su color, sabor y poder de expansión. Como poder de expansión se entiende la capacidad de crecimiento durante el horneado de una masa preparada con el almidón.

Esta investigación tiene el objetivo de analizar cómo se puede llegar a un estándar en el poder de expansión deseado del almidón agrio de yuca, mezclando raíces de yuca o almidones de 
diferentes calidades. Se muestra que al procesar juntamente raíces de variedades con alto y con bajo poder de expansión, se obtiene un almidón con una calidad intermedia. Así mismo, al mezclar almidones agrios de diferentes calidades se llega a un producto con un poder de expansión entre aquel de las componentes que entran en la mezcla.

El análisis se basa en modelos estadísticos de superficie de respuesta, que describen el resultado de la mezcla de varias componentes en términos de las proporciones usadas. Los datos para la experimentación provienen de raíces de yuca del Morte del Cauca. Los almidones se obtienen con la tecnología de producción tradicional en plantas de esta zona.

Los resultados del estudio permiten concluir que el rallandero puede ajustar la calidad de sus almidones por procesos de mezcla. Tanto la mezcla de raíces como la mezcla de almidones son controlables. De esta manera, el rallandero tiene la posibilidad de aprovechar ofertas favorables de raíces y ajustarse a las condiciones variables del mercado de almidón agrio.

Palabras Clave: Agroindustria rural, almidón agrio, yuca, poder de expansión, modelo de superficie de respuesta.

\section{ABSTRACT}

The cassava sour starch is obtained manually by natural fermentation of native starch. It is employed in the elaboration of traditional bakery products. Its expected properties refer to its color, taste and expansion power. Expansion power means the growing capability during the baking process of some dough prepared with starch.

The present research intents to analyze how to reach a given expansion power standard for cassava sour starch, by mixing cassava roots or starches of different quality levels. It is shown that mixing roots from cassava varieties of high and low expansion powers an intermediate quality starch is obtained. In the same way, mixing starches of different power expansions, one obtains starch of a quality ranked between the components of the mixture.

The analysis is based on statistic surface response models that describe the results of mixing various components as a function of the proportions used. The experimentation data originate from roots harvested in the Morth of the Cauca Department. Starches are obtained by the traditional production technology applied in the small local factories.

The outcomings permit to conclude that the starch manufacturer can adjust the starch qualities by a mixing process. Mixing roots as well as mixing starches are controllable processes. Thus, the starch manufacturer is able to take advantage of profitable offers and to adjust to changing starch market conditions.

Key Words: Rural agroindustry, sour starch, cassava, specific volume, surface response model.

\section{INTRODUCCIÓN}

\section{Justificación:}

Uno de los conceptos importantes de la filosofía de mejoramiento continuo que ya mostró indudablemente su poder, es el de calidad total. El empresario debe ofrecer a su cliente el producto en el momento que éste lo requiere, en la cantidad que el cliente necesita y con las especificaciones definidas por el cliente mismo. Sólo el cumplimiento con la calidad en estos tres aspectos puede crear el ambiente de mutua confiabilidad, que es deseable en la relación empresa - cliente.

El productor del almidón de yuca, considerado como gerente de una pequeña o mediana empresa, se debe ajustar también a estos conceptos importantes. La calidad del almidón no depende solamente del proceso productivo que él mismo controla. La variedad de yuca, la madurez de las raíces, el tiempo entre cosecha y procesamiento de la yuca, el tiempo de almacenamiento del almidón y la calidad del agua 
Normalización de la Calidad del Almidón Agrio de Yuca, a través de Mezclas de Almidones de Raíces de Yuca.

usada en el proceso son otros factores de un alto impacto en la calidad, difícilmente modificables por el productor. Por esta razón se obtienen almidones de una amplia gama de calidades. El productor necesita mecanismos de normalización que permiten modificar la calidad de los almidones para poder ofrecer a sus clientes el almidón de acuerdo con los estándares que ellos piden.

La presente investigación tiene el objetivo de analizar cómo se puede llegar a un estándar de calidad deseado, mezclando raíces de yuca o almidones de diferentes calidades. Se espera que al procesar juntamente raíces de variedades buenas y malas en cuanto a su poder de expansión, se obtenga un almidón con una calidad intermedia. Así mismo, al mezclar almidones agrios de diferentes calidades se quiere llegar a un producto con un poder de expansión entre aquel de las componentes que entran en la mezcla.

Ajustando por mezcla de raíces o de almidones la calidad del producto final, el rallandero podría obtener las siguientes ventajas:

1. Pude aprovechar una oferta de raíces baratas, ya que con una cantidad determinada de yuca de alto poder de expansión obtendrá un producto con una calidad aceptada en el mercado.

2. Los almidones de poco poder de expansión, que no tienen demanda y consecuentemente deben venderse a un precio bajo, pueden llegar a clasificarse en una categoría de precios mejor, si se agrega almidón de calidad superior en una proporción determinada.

3. La posibilidad de manejar los niveles de calidad por mezcla, permite al rallandero reducir la cantidad de almidón agrio almacenada.

4. Le da al rallandero la flexibilidad de ajustarse a los requerimientos de un mercado cambiante, ofreciendo a cada cliente el almidón en la calidad deseada.

\section{El proceso productivo del almidón agrio de yuca:}

El almidón agrio de yuca es utilizado en la elaboración de productos de panadería como los pandebonos, pandeyucas y snacks. Las características solicitadas por la industria son su color, su sabor y su poder de expansión. El almidón debe ser bien blanco, libre de impurezas. Se busca el aroma agrio, que confiere el sabor típico al producto final. Para la panificación es importante que la masa tenga un buen crecimiento. El poder de expansión se define como el cociente entre volumen y peso de una masa después del proceso de horneado. Estas propiedades se logran, exponiendo un almidón nativo a un proceso de fermentación natural y de secado al sol.

La producción del almidón es una agroindustria tradicional en Colombia. Aproximadamente el $80 \%$ de la producción nacional se procesa en plantas ubicadas en el Departamento del Cauca, conocidas como rallanderías, con capacidades productivas que oscilan entre 1 y 5 toneladas de raíces por día de acuerdo a su grado de mecanización. El almidón, con un 90\% de contenido en base seca, es el principal constituyente de la raíz de yuca. El proceso productivo consiste en extraer el almidón de la raíz de yuca por vía húmeda.

El proceso tradicional para la producción de almidón consta de las siguientes operaciones: Las raíces de yuca se lavan para eliminar las impurezas con las que vienen del campo; adicionalmente se desprende en el proceso de lavado la cascarilla o corteza exterior de la raíz que es muy delgada y sale fácilmente por fricción. La yuca lavada se ralla para desintegrar al máximo las células de la raíz que contienen en su interior el almidón. La masa rallada se mezcla con agua y se pasa por un filtro, para separar las fibras del almidón que sale disuelto en el agua. La sedimentación es el proceso de separar el almidón del agua por precipitación en tanques o canales de sedimentación. El almidón se 
fermenta en tanques entre 20 a 50 días. Las bacterias lácticas responsables para la fermentación modifican el almidón y producen el ácido láctico que le da el sabor característico (Jory, 1989; Cárdenas et al., 1980). El secado se realiza, exponiendo el almidón a los rayos solares hasta obtener humedades del 10 al 13\%. Los rayos ultravioletas, actuando sobre el almidón húmedo fermentado son indispensables para que el almidón adquiera su poder de expansión (Asté, 1995).

\section{Mezcla de raíces:}

La mezcla de raíces de diferentes variedades es una práctica común entre los rallanderos. Como la oferta de raíces es fluctuante, el rallandero tiene que aceptar a veces lotes pequeños. De esta forma se llega a mezclar raíces de diferentes calidades. En otras ocasiones, el rallandero puede negociar un lote grande de raíces, pero de una variedad que según su experiencia produce almidones de mala calidad. En esta situación, el rallandero busca refinar su producto final, comprando una pequeña cantidad de raíces de variedades que prometen un buen poder de expansión. Mo utiliza criterios cuantitativos para determinar en qué proporción usar las diferentes raíces, se basa en su experiencia e intuición.

Una búsqueda bibliográfica exhaustiva mostró que la mezcla de raíces de yuca para mejorar la calidad del almidón agrio no ha sido hasta el momento tema de investigación científica publicada en los medios. En otras palabras, no se conocen estudios cuantitativos que relacionen el poder de expansión de una mezcla de raíces de yuca con el poder que alcanzan las variedades puras. En este sentido, la presente publicación puede considerarse un estudio piloto y una invitación a otros investigadores a continuar el trabajo sobre esta temática.

\section{Mezcla de almidones:}

El rallandero en el sur occidente de Colombia no conoce la práctica de mezclar almidones para ajustar su calidad, posiblemente por falta de un método confiable y de reconocimiento general, que le permita medir el efecto de la mezcla de productos. En cambio, los consumidores del almidón sí producen según sus propios criterios, mezclas de almidones para obtener la materia prima más adecuada para cada uno de sus productos.

Un estudio sistemático cuantitativo sobre la problemática se realizó en una investigación realizada en la Universidad del Valle (Tischer et al., 1999), donde se considera la mezcla de almidones, como uno de diferentes aspectos que influyen en la calidad del almidón agrio. Junto con estudiantes de Estadística en la Universidad del Valle que desarrollaron su proyecto de grado en el marco de esta investigación (Mosquera et al., 1998), se iniciaron pruebas preliminares y se formuló un modelo de regresión tratando de comprobar que al mezclar dos calidades de almidones en igual proporción, se puede lograr un producto homogéneo, con calidad intermedia que pueda ser vendido por el rallandero a un precio comercial. Este proyecto constituye hasta el momento el único antecedente en la temática, así que puede ser considerado un estudio piloto para la presente investigación, que pretende desarrollar la temática con más generalidad.

\section{METODOLOGÍA}

\section{Método para la evaluación del poder de expansión:}

Esencial para los experimentos que se describen a continuación, es la evaluación del poder de expansión de un almidón agrio de yuca. La prueba usada se describe a continuación: Una mezcla de $10 \mathrm{gr}$ de almidón con $12 \mathrm{~cm}^{3}$ de agua, se hornea por 22 min a $450^{\circ} \mathrm{F}$. La mezcla crece y forma un panecillo. Éste se deja enfriar a temperatura ambiente y luego se mide su volumen y peso. Se determina el cociente entre volumen y peso para obtener el poder de expansión (Fernández, 1999). 
Normalización de la Calidad del Almidón Agrio de Yuca, a través de Mezclas de Almidones de Raíces de Yuca.

\section{El modelo de mezclas:}

El modelo estadístico más apropiado para representar las mezclas de raíces de yuca y de almidones es el modelo de superficie de respuesta. Se puede considerar el modelo como forma cuadrática, donde se incluye una interacción entre las componentes de la mezcla, o como función lineal, que no considera interacción(Montgomery, 1991). El modelo describe el poder de expansión de la mezcla en términos de las proporciones con las cuales se mezclan las componentes:

\begin{tabular}{|c|}
\hline \multicolumn{2}{|c|}{ Modelo sin interacción } \\
\hline$Y_{k}=\sum_{i=1}^{\text {imax }} \beta_{i} x_{i k ;}$ \\
$k=1,2, \ldots$, , $\max ;$
\end{tabular}

\begin{tabular}{|c|}
\hline$y_{k}=\sum_{i=1}^{i m a x} \beta_{i} x_{i k}+\sum_{\substack{i, j=1 \\
i \neq j}}^{i m a x} \beta_{i j} x_{i k} x_{j k}$ \\
$k=1,2, \ldots, k \operatorname{kmax} ;$
\end{tabular}

$0 \leq x i k \leq 1$;

$i=1,2, \ldots$, imax $;=1,2, \ldots, k \max$

$\sum_{i=1}^{i m a x} x i k=1 ; \quad k=1,2, \ldots, k \max$;

Donde:

$i, j \quad$ se refiere a las componentes (variedades de yuca o almidones agrios respectivamente), que entran en el modelo de mezclas;

imax es el número de componentes considerados en el modelo;

$i, j=1,2, \ldots$, imax.

$k \quad$ identifica la observación (una mezcla);

kmax es el número de observaciones realizadas; $k=1,2, \ldots, k \max$;

$y_{k} \quad$ es el poder de expansión resultante de la mezcla $k$ de las componentes;

$x_{i k} \quad$ es la proporción de la $i$-ésima componente en la observación $k$;

$i=1,2, \ldots$, imax $; k=1,2, \ldots$, kmax;
$\mathrm{B}_{i} \quad$ representa la respuesta esperada correspondiente a la $i$-ésima componente;

$\beta_{i j} \quad$ representa el efecto correspondiente al producto cruzado al mezclar la componente $i$ con la componente $j$; $i, j=1,2, \ldots$, imax

Experimentación para las mezclas de raíces:

La experimentación de las mezclas de raíces de yuca se llevó a cabo en la rallandería la Agustina, Departamento de Cauca, entre diciembre de 1998 y febrero de 1999. El almidón resultante se llevó a la Planta Piloto de Procesamiento de Alimentos en la Universidad del Valle, donde se realizaron las pruebas de expansión.

El experimento consiste en evaluar las mezclas de raíces de dos variedades de yuca en proporciones determinadas y relacionar el resultado con la calidad de las raíces sin mezclar. Se realizaron 2 experimentos, cada uno con dos réplicas usando raíces pertenecientes al mismo lote de yuca, cosechadas en la misma semana. Las variedades de yuca usadas fueron Algodona y Parra en el primer experimento y Algodona y Amarga en el segundo.

El procedimiento fue el siguiente:

Para cada mezcla de dos variedades de yuca se usó $160 \mathrm{~kg}$ de yuca en total, repartidos según las siguientes proporciones: 100:0, 75:25, 50:50, 25:75, 0:100.

Las raíces mezcladas se procesaron, aplicando los equipos y procedimientos habituales en la rallandería. La fermentación del almidón se realizó en tubos de PVC, usando 2 tubos para cada mezcla, cada uno con aproximadamente $10 \mathrm{~kg}$ de almidón. Los almidones fermentados se secaron al sol, usando 2 kg de almidón de cada tubo. Todas las mezclas de las 2 réplicas se secaron el mismo día, para minimizar la influencia climática.

Para controlar el proceso de producción, se midieron las siguientes variables: la cantidad de almidón húmedo resultante de cada mezcla, la cantidad de almidón en cada tubo, el almidón 
seco resultante de $2 \mathrm{~kg}$ de almidón fermentado y el porcentaje de humedad del almidón seco. Posteriormente se determinó en el laboratorio el poder de expansión de cada muestra. Se aplica y evalúa el modelo de mezclas a los resultados obtenidos.

\section{Experimentación para las mezclas de almidones:}

El experimento consiste en la evaluación de la relación que existe entre el poder de expansión de una mezcla de almidones en proporciones determinadas y el poder de expansión de los almidones que forman parte de la mezcla. Se desarrolla en las siguientes fases:

Se decidió usar tres categorías de calidad de almidón, de acuerdo con su poder de expansión: malo ( $\left.\leq 8 \mathrm{~cm}^{3} / \mathrm{gr}\right)$; regular $\left(8-11 \mathrm{~cm}^{3} / \mathrm{gr}\right.$ ) y bueno $\left(>11 \mathrm{~cm}^{3} / \mathrm{gr}\right)$. Se seleccionan 3 almidones de cada una de las 3 categorías, provenientes de las rallanderías regionales. De cada almidón se separa la cantidad suficiente para la experimentación y se registra su poder de expansión. Para cada posible combinación de 2 almidones se realizan mezclas en las proporciones $75: 25,50: 50,25: 75$ y se evalúa el poder de expansión de las mezclas.

Se aplica y evalúa el modelo de mezclas a los resultados obtenidos. Como se quiere usar el modelo para predecir el comportamiento de una mezcla cualquiera de dos almidones, es necesario evaluar el poder predictivo del modelo: se aplica el experimento de mezclas a 3 almidones seleccionados aleatoriamente, uno de cada categoría. Se analiza la diferencia entre el valor observado en la experimentación y el valor estimado con el modelo con el fin de determinar el error de estimación y la bondad de ajuste del modelo.

\section{RESULTADOS Y DISCUSIÓN PARA LA MEZCLA DE RAÍCES}

\section{Los datos obtenidos}

El proceso productivo no varía durante toda la experimentación, las variables de control toman valores con diferencias insignificantes. El rendimiento se puede considerar igual en todos los tubos del experimento. Pequeñas variaciones se pueden explicar por imprecisiones que se deben al manejo de una gran cantidad de raíces de la cual resulta relativamente poco almidón seco $(160 \mathrm{~kg}$ de raíces produjeron entre 40 y 60 kg de almidón fermentado, y por cada 2 kg de éste se obtuvo alrededor de $1200 \mathrm{gr}$ de almidón seco). Las pruebas de humedad permiten acertar que todos los almidones llegaron a humedades óptimas en cuanto a almacenamiento del almidón (entre 11 y 14\% de humedad).

Las pruebas de calidad dentro de un tubo arrojan valores muy similares, el coeficiente de variación que se obtuvo en los 40 tubos de los diferentes experimentos está por debajo de $11 \%$ en el $90 \%$ de las observaciones. Por lo general, el poder de expansión medido en los 2 tubos correspondientes a una mezcla de raíces puede considerarse igual. Esto justifica el hecho de que se seleccionó el poder de expansión promedio entre los 2 tubos como variable de análisis. Se obtuvieron los siguientes resultados:

\begin{tabular}{|c|c|c|c|c|}
\hline & \multicolumn{2}{|c|}{$\begin{array}{c}\text { Experimento 1 } \\
\text { Algodona - Parra }\end{array}$} & \multicolumn{2}{c|}{$\begin{array}{c}\text { Experimento 2 } \\
\text { Algodona - Amarga }\end{array}$} \\
\hline & Réplica 1 & Réplica 2 & Réplica 1 & Réplica 2 \\
\hline $100-0$ & 10.43 & 8.96 & 10.41 & 9.96 \\
$75-25$ & 10.34 & 9.39 & 10.47 & 8.92 \\
$50-50$ & 9.74 & 8.73 & 6.98 & 8.22 \\
$25-75$ & 7.71 & 9.51 & 6.17 & 9.33 \\
$0-100$ & 5.30 & 5.80 & 6.99 & 7.63 \\
\hline
\end{tabular}

Tabla 1. Resultados de los dos experimentos de mezcla de raíces. La variable de respuesta es el poder de expansión, promediado sobre los 2 tubos de cada muestra.

Para visualizar los resultados, se ordenaron los datos según la proporción, empezando con la mejor componente (figura 1). Se observa una tendencia a bajar la calidad al disminuir la proporción de las raíces de mejor calidad. 
Normalización de la Calidad del Almidón Agrio de Yuca, a través de Mezclas de Almidones de Raíces de Yuca.

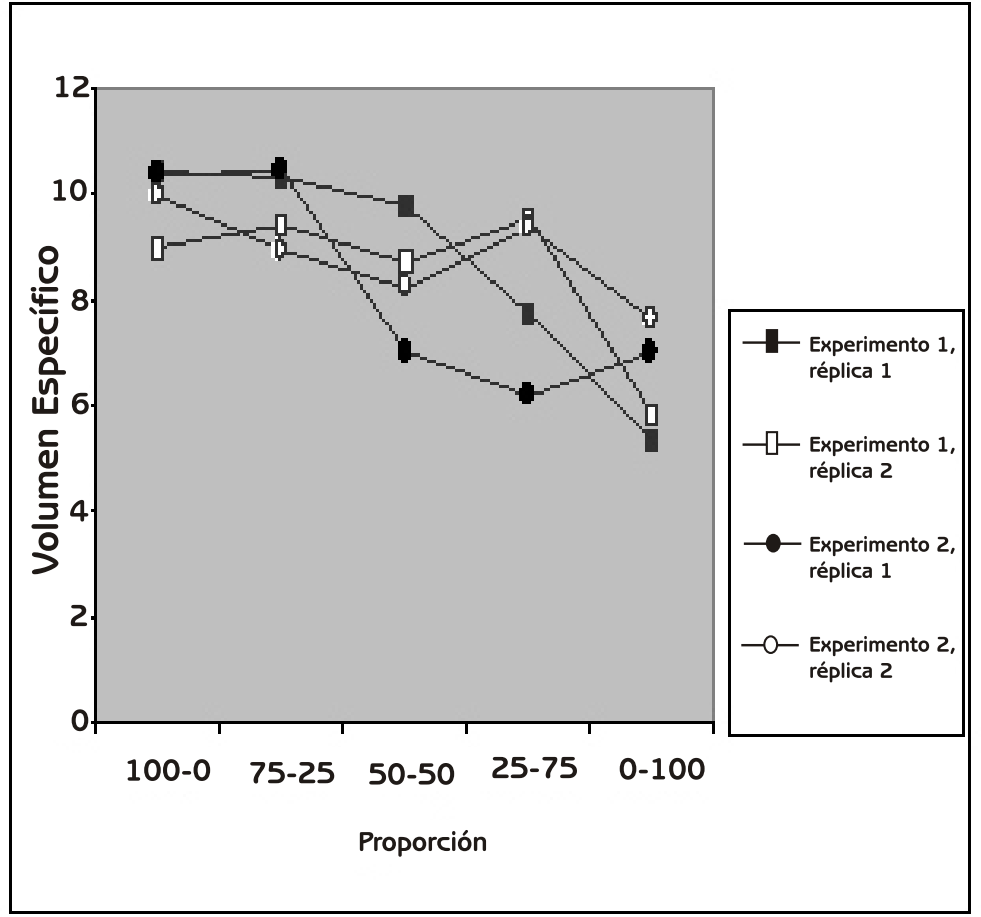

Figura 1. El poder de expansión de la mezcla de raíces. Promedio de los dos tubos correspondientes a cada mezcla

\section{Modelación:}

El modelo de mezcla (con y sin interacción de las componentes) se aplicó en dos escenarios para determinar aquel que se ajuste mejor a los resultados obtenidos.

El modelo por variedades considera las tres variedades de yuca, que se usó en la experimentación: Algodona, Parra y Amarga. Inicialmente se aplicó este modelo a todos los datos obtenidos, luego se eliminaron dos puntos outliers.

El modelo por categorías usa la clasificación de las variedades de acuerdo a su poder de expansión, como se lo fijó para los almidones en el experimento de mezclas: malo, regular y bueno. Según esta clasificación, el presente experimento considera mezclas entre variedades de calidad regular y mala. También se aplicó este modelo primero al conjunto completo de datos, eliminando después dos outliers.

En ambos modelos se estudió en las variantes con y sin interacción entre las componentes de la mezcla.

La tabla 2 resume los resultados de la modelación.

\section{Discusión:}

Minguno de los modelos que usan los datos completos muestra un ajuste muy bueno. Una posible explicación para este resultado puede ser que el período de fermentación se prolongó (43 y 34 días) porque condiciones climáticas desfavorables no permitían secar el almidón. Según una investigación reciente, una fermentación prolongada tiende a aumentar la variabilidad del poder de expansión de un almidón (Tischer et al., 1999). La precisión podría ser afectada también por el hecho de que se manipula una gran cantidad de materiales (yuca y almidón), bajo condiciones habituales en la rallandería.

Eliminando dos outliers, el ajuste mejora considerablemente, razón por la cual se limita al análisis de estos modelos.

Para todos los modelos que incluyen interacción entre las componentes de la mezcla se obtiene que la interacción no es significativa (niveles altos de significancia), lo que justifica usar el modelo sin interacción para el análisis.

Dado que los valores de los parámetros del modelo por variedades $(10.5,6.2,6.8)$ se acercan a la calidad de la variedad de yuca correspondiente (algodona 10.3; parra 5.5, amarga 7.3), se puede concluir que el poder de expansión de una mezcla de raíces de dos variedades depende sólo de la calidad de las componentes y de la proporción.

Puesto que el rallandero no conoce con anticipación el poder de expansión de una variedad sino después del proceso productivo, el 
anterior modelo es poco operativo, razón por la cual se recomienda más el modelo por categorías. A partir de su experiencia, el rallandero probablemente pude estimar bien si las raíces que tiene a su disposición son de calidad buena, regular o mala.

El modelo por categorías sin interacción es ligeramente mejor que el anterior. Es muy sencillo y fácil de interpretar: El poder de expansión de la mezcla entre variedades con calidad regular y mala corresponde a la proporción, ponderada con los parámetros respectivos de cada categoría. Los valores de estos parámetros (10.5 y 6.6) representan el poder de expansión típico de las categorías respectivas. Se acercan mucho a los valores de los promedios de las variedades en cada categoría (10.3 y 6.4).

\begin{tabular}{|l|c|l|c|}
\hline \multicolumn{1}{|c|}{ Modelo } & $\begin{array}{c}\text { Elim. } \\
\text { Outliers }\end{array}$ & \multicolumn{1}{|c|}{ Resultado } & $\mathbf{R}^{2}$ \\
\hline Modelo por variedades con interacción & no & $y=9.6 x_{1}+5.8 x_{2}+7.2 x_{3}+7.2 x_{1} x_{2}-0.8 x_{1} x_{3}$ & 0.46 \\
Modelo por variedades sin interacción & no & $y=10.0 x_{1}+6.8 x_{2}+7.0 x_{3}$ & 0.36 \\
Modelo por categorías con interacción & no & $y=9.6 x_{1}+6.5 x_{2}+3.2 x_{1} x_{2}$ & 0.41 \\
Modelo por categorías sin interacción & no & $y=10.0 x_{1}+6.69 x_{2}$ & 0.40 \\
Modelo por variedades con interacción & sí & $y=10.4 x_{1}+5.6 x_{2}+7.3 x_{3}+4.7 x_{1} x_{2}-2.6 x_{1} x_{3}$ & 0.66 \\
Modelo por variedades sin interacción & sí & $y=10.5 x_{1}+6.2 x_{2}+6.8 x_{3}$ & 0.60 \\
Modelo por categorías con interacción & sí & $y=10.4 x_{1}+6.4 x_{2}+1.0 x_{1} x_{2}$ & 0.59 \\
Modelo por categorías sin interacción & sí & $y=10.5 x_{1}+6.6 x_{2}$ & 0.61 \\
\hline
\end{tabular}

Tabla 2. Resultados de la modelación. La variable de respuesta y es el poder de expansión, x se refiere a la proporción de la i-ésima componente en la mezcla (variedad o categoría).

Antes de plantear conclusiones estrictas es necesario repetir el experimento a mayor escala y con más variedades de yuca para obtener resultados también para mezclas entre las categorías bueno - regular y bueno - malo.

A partir de los resultados presentes se puede concluir que el rallandero sí puede influir sobre la calidad de un almidón por mezclar diferentes raíces. Una yuca de buena calidad estará en capacidad de aumentar la calidad de un almidón de inferior calidad.

\section{RESULTADOS Y DISCUSIÓN PARA MEZCLA DE ALMIDONES}

\section{Los datos obtenidos:}

Se seleccionaron 3 almidones de cada una de las categorías malo, regular y bueno y se realizaron las mezclas en las proporciones establecidas. Tanto en los almidones puros como en los mezclados, la variación intramuestral era muy baja. Se eliminaron 3 valores para obtener un coeficiente de variación por debajo de 10\% en todas las observaciones intramuestrales. Los resultados de la evaluación del poder de expansión se resumen en la tabla 3.

Se observa que en mezclas de una categoría baja con otra mejor, se aumenta el poder de expansión resultante en la medida en que crece la proporción del mejor. La modelación confirma este resultado. Para dar una idea de cómo se comportan las mezclas, se muestra en la figura 2 el promedio de las mezclas de entre las categorías malo - malo, malo - regular, malo bueno, regular - regular, regular - bueno y bueno bueno, según la proporción de la mezcla (en la modelación no se usan los promedios, sino todos los datos obtenidos).

\section{Modelación:}

Al igual que en la experimentación con mezclas de raíces, se aplicaron los modelos de mezclas 
Normalización de la Calidad del Almidón Agrio de Yuca, a través de Mezclas de Almidones de Raíces de Yuca.

\begin{tabular}{|c|c|c|}
\hline Categoría & Almidón & Poder de expansión \\
\hline malo & 1 & 6.95 \\
& 2 & 7.57 \\
regular & 3 & 6.52 \\
& 4 & 12.14 \\
& 5 & 10.96 \\
bueno & 6 & 10.31 \\
& 7 & 13.84 \\
& 8 & 13.38 \\
& 9 & 13.11 \\
\hline
\end{tabular}

Tabla 3. El poder de expansión de los almidones seleccionados con y sin interacción entre las componentes. El resultado se resume en la tabla 4.

El modelo de mezclas con interacción tuvo un buen ajuste (69\%), indicando que el poder de expansión puede ser expresado en dependencia de las componentes puras que entran a la mezcla y de una interacción entre los grupos de almidones. En términos generales: se puede usar la mezcla de almidones para ajustarse a la calidad que exige el cliente.

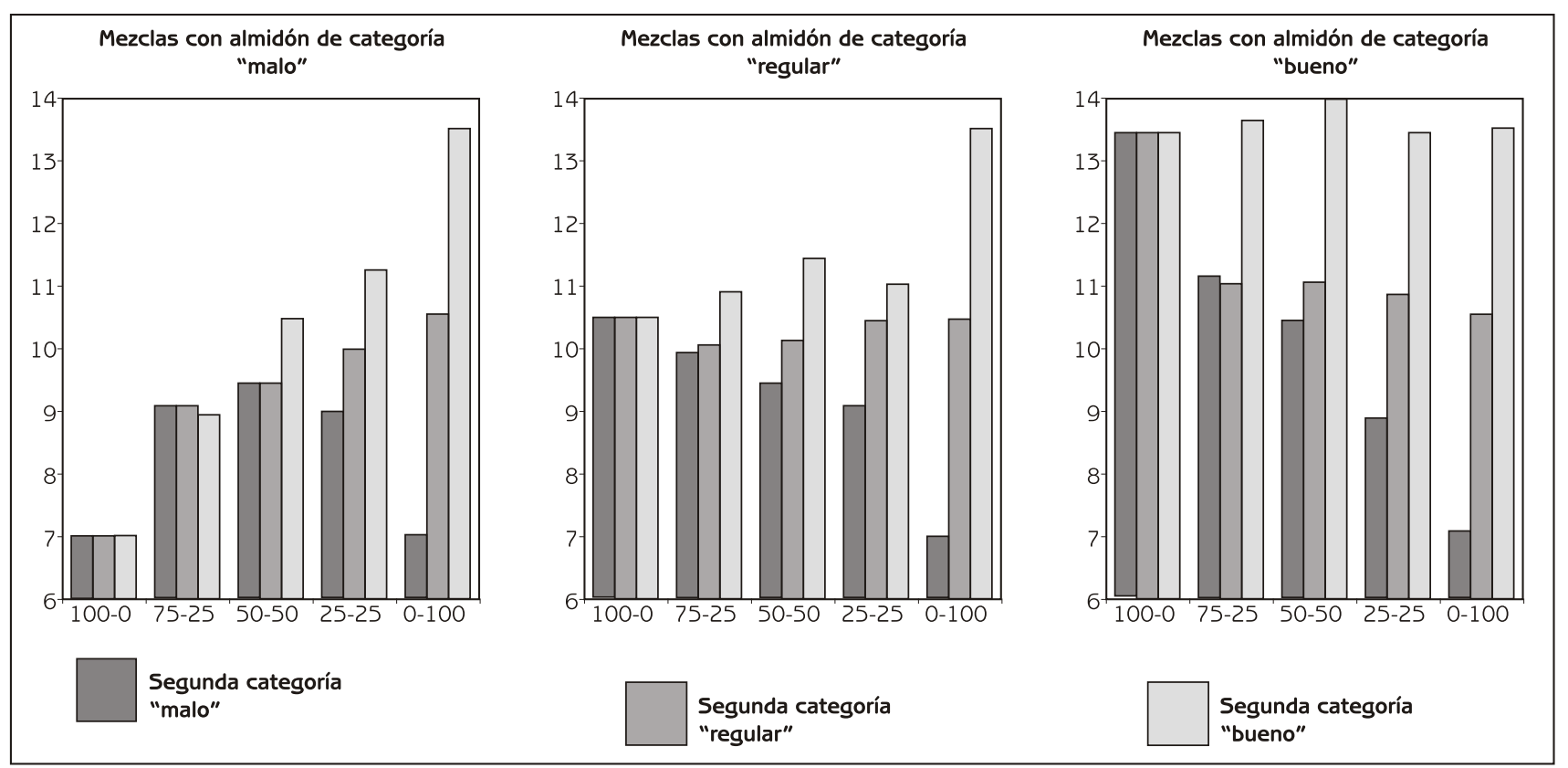

Figura 2. Comportamiento de mezclas de almidones entre categorías

\begin{tabular}{|l|c|c|}
\hline \multicolumn{1}{|c|}{ Modelo } & \multicolumn{1}{|c|}{ Resultado } & $\mathbf{R}^{2}$ \\
\hline $\begin{array}{l}\text { Modelo por categorías con interacción } \\
\text { Modelo por categorías sin interacción }\end{array}$ & $\begin{array}{l}y=8.6 x_{1}+10.5 x_{2}+13.3 x_{3}-0.3 x_{1} x_{2}-3.4 x_{1} x_{3}-4.0 x_{2} x_{3} \\
0.69\end{array}$ & 0.65 \\
\hline
\end{tabular}

Tabla 4. Resultados de la modelación. La variable de respuesta y es el poder de expansión, xi se refiere a la proporción de la i-ésima categoría en la mezcla (1: malo; 2: regular; 3: bueno).

El coeficiente correspondiente a la interacción del grupo de almidones malos con regulares no aporta significativamente al modelo $(p=0.8)$, es decir, no hay interacción entre la categoría mala y regular. Los coeficientes correspondientes a la interacción con el grupo de almidones buenos (malo - bueno, regular- bueno) son negativos.
Esto quiere decir que una mezcla con almidones buenos no arroja los resultados que se esperaría suponiendo un modelo lineal. Los almidones buenos sí mejoran la calidad de la mezcla, pero no tienen un efecto tan positivo como lo esperado.

Se analizó también el modelo lineal, dado que es 
muy intuitivo y de fácil implementación para el rallandero, ya que no es necesario tener en cuenta posibles interacciones entre grupos de almidones. Como es de esperar, el coeficiente de determinación baja, pero muy ligero (de $69 \%$ a 65\%). El modelo que no supone interacción tiene un buen ajuste, cada grupo de almidones aporta significativamente (niveles de significancia prácticamente 0) a la explicación del poder de expansión. Estos resultados justifican la aplicación del modelo sin interacción.

\section{Poder predictivo de los modelos}

Se evaluaron las mezclas de tres almidones adicionales, uno de cada categoría. Se contrastó mediante una prueba $x^{2}$ el poder de expansión obtenido para una mezcla con el valor teórico que arroja el modelo. Las calidades de los almidones son: almidón malo: 6.31; almidón regular: 9.74; almidón bueno: 12.28. En la siguiente tabla se muestran los valores observados con la predicción de los modelos con y sin interacción. En ambos casos, se confirma que lo predicho con el modelo coincide con la observación real, con una probabilidad que es prácticamente 1 .

\section{CONCLUSIONES}

Las categorías del almidón agrio basadas en esta prueba todavía no se han estandarizado. La presente experimentación surgiere una clasificación en malo $\left(<8 \mathrm{~cm}^{3} / \mathrm{gr}\right)$, regular (8-11 $\mathrm{cm}^{3} / \mathrm{gr}$ ) y bueno $\left(>11 \mathrm{~cm}^{3} / \mathrm{gr}\right)$. Se recomienda someter estas categorías a una discusión con los usuarios de la prueba.

\begin{tabular}{|l|l|l|l|l|l|l|}
\hline & \multicolumn{1}{|c|}{ Mezcla } & $\mathbf{1 0 0}$ & $\mathbf{7 5}$ & $\mathbf{5 0}$ & $\mathbf{2 5}$ & \multicolumn{1}{c|}{$\mathbf{0}$} \\
\hline \multirow{2}{*}{ Observación } & malo - regular & 6.31 & 7.75 & 8.42 & 8.71 & 9.74 \\
& malo - bueno & 6.31 & 8.40 & 9.55 & 11.44 & 12.28 \\
& regular - bueno & 9.74 & 10.78 & 11.52 & 12.60 & 12.28 \\
& & & & & & \\
\hline \multirow{2}{*}{ Modelo con interacción } & malo - regular & - & 9.02 & 9.48 & 9.98 & - \\
& malo - bueno & - & 9.77 & 10.94 & 12.12 & - \\
& regular - bueno & - & 10.47 & 10.92 & 11.86 & - \\
\hline Modelo sin interacción & malo - regular & - & 8.87 & 9.33 & 9.80 & - \\
& malo - bueno & - & 9.47 & 10.52 & 11.58 & - \\
& regular - bueno & - & 10.85 & 11.45 & 12.04 & - \\
\hline
\end{tabular}

Tabla 5. El poder predictivo de los modelos de mezcla de almidones

El modelo de mezclas era la herramienta más adecuada para la experimentación con raíces y con almidones. Se pudieron aplicar variantes de este modelo para analizar mezclas de raíces y de almidones.

El experimento de mezclas de raíces se realizó como prueba piloto, dado que no existe hasta el momento ningún estudio cuantitativo sobre el comportamiento de mezclas de raíces. Se pudo mostrar que al mezclar dos variedades de yuca se obtiene un almidón de poder de expansión intermedio que corresponde a la proporción de la mezcla.
El rallandero no conoce con anticipación la calidad de las variedades de yuca que procesa, pero sí las puede clasificar en las categorías "bueno", "regular" y "malo". Por esta razón se interpretaron los resultados de la experimentación también usando estas categorías. Como las raíces usadas se clasificaron como regulares y malos, se puede concluir a partir de la presente experimentación, que al mezclar raíces de categoría "malo" con otras de categoría "regular", se puede esperar un poder de expansión que depende linealmente de la proporción de la mezcla.

Para tener más claridad sobre el comportamiento 
de mezclas de raíces es necesario realizar experimentos adicionales, con el fin de obtener más información sobre el comportamiento de las combinaciones entre variedades con las clasificaciones "malo" y "regular" y de obtener las bases para analizar las combinaciones de tipo "malo - bueno" y "regular - bueno". Además falta validar el poder de predicción de los modelos en este contexto.

La investigación afirmó cuantitativamente la operacionalidad de una práctica tradicional en la rallandería: se puede mejorar el poder de expansión esperado de un almidón, si se procesa la yuca con mala perspectiva junto con otra variedad que promete mejor calidad.

Para el experimento con mezclas de almidones agrios se pudo hacer referencia a un ensayo anterior. La presente investigación logró confirmar y ampliar los resultados del estudio anterior. Las mezclas de almidones se analizaron usando modelos de mezcla con y sin interacción entre las componentes. Ambos modelos mostraron un buen ajuste. La evaluación del poder predictivo de los modelos afirma que ambos pueden ser utilizados para predecir el poder de expansión de una mezcla de almidones agrios en dependencia de las proporciones a usar. Alternamente se puede, conociendo el poder de expansión de una serie de almidones agrios, determinar la proporción precisa para lograr una calidad determinada. De esta manera, los rallanderos, y por supuesto los intermediarios y consumidores finales, pueden usar almidones que tienen a su disposición para lograr el poder de expansión que se requiere en un momento dado.

Resumiendo, se puede decir que existe la posibilidad de ajustar el poder de expansión de almidones agrios por procesos de mezcla. Tanto la mezcla de raíces como la mezcla de almidones son controlables. Una vez operacionalizados los resultados de esta investigación, el rallandero puede aprovechar de ofertas de raíces y ajustarse a los requerimientos específicos del mercado de almidón agrio.

\section{BIBLIOGRAFÍA}

1. Asté M., 1995. Contribution à l'étude de l'acquisition du pouvoir de panification de l'amidon aigre de manioc: rôles de l'acide lactique et du rayonnement solaire. Mémoire de DEA en Sciences Alimentaires et Assurance Qualité de I'Institut des Sciences et Techniques des Aliments de Bordeaux (ISTAB), 49 p., CIRAD/CIAT/, Cali, Colombia.

2. Cárdenas O.S, de Buckle T.S., 1980. Sour cassava starch production: A preliminary study. Journal of Food Science. Vol. 45.

3. Fernández, A.; Zakhia M. 1999. Desarrollo de un método sencillo para medir la calidad del Almidón agrio de yuca. Impacto del método sobre la agroindustria rural en el norte del departamento del Cauca, Colombia. Seminario Agroindustria Rural y Globalización frente al próximo milenio. Quito, Ecuador.

4. Jory M. 1989. Contribution a l'etude de deux processus de transformation du manioc comportant une phase de fermentation. Rapport de Mastere, Doc. Ceemat. Montpellier, Francia.

5. Montgomery, Douglas. 1991. Diseño de experimentos. Grupo Editorial iberoamericana.

6. Mosquera, A.; Ortega, J.L. 1998. Un modelo de regresión para predecir la calidad de mezclas de almidones agrios de yuca. Proyecto de grado, Programa Académico de Estadística, Universidad del Valle.

7. Tischer, I.; Andrade, M.; Moreno M.; 1999. Mejoramiento de la productividad y de la calidad de la rallandería de yuca. Reporte final de investigación. Universidad del Valle, Cali. 